\title{
Ischemic Stroke Caused by Paradoxical Embolism After an Unsuccessful Transcatheter Atrial Septal Defect Closure Procedure: A Word of Caution
}

\author{
Zhen-fei Fang $\cdot$ Liang Tang $\cdot$ Sheng-hua Zhou
}

Received: 9 April 2011/Accepted: 15 June 2011/Published online: 27 November 2011

(C) The Author(s) 2011. This article is published with open access at Springerlink.com

\begin{abstract}
Transcatheter device closure of atrial septal defect (ASD) has become a well-accepted alternative to surgical repair. Serious complications of transcatheter ASD closure are rare, but when they occur, devastating consequences may result. Herein, we present the case of a 4-year-old girl who had an ischemic stroke caused by a presumptive paradoxical embolism after an unsuccessful transcatheter ASD procedure and in whom subsequent venous color Doppler showed deep venous thrombosis (DVT) of the right lower extremity. The risk factors that predisposed to paradoxical cerebral embolism and DVT in this patient are discussed, and the literature is reviewed.
\end{abstract}

Keywords Paradoxical embolism - Atrial septal defect . Deep venous thrombosis

\section{Introduction}

Atrial septal defect (ASD) is one of the most common forms of congenital heart disease, accounting for approximately $10 \%$ of all congenital cardiac defects [8]. Transcatheter device closure of secundum ASD has become a wellestablished alternative to open heart surgery in appropriate patients. The procedure is associated with a high success rate and favorable short- and long-term results [10]. Despite this fact, some catastrophic complications, including systemic embolization, complete atrioventricular block, and cardiac perforation, have been known to occur during the

Z. Fang $\cdot$ L. Tang $\cdot$ S. Zhou $(\bowtie)$

Department of Cardiology, The Second Xiangya Hospital of Central South University, Changsha, Hunan 410011, China

e-mail: zhougqin@21cn.com perioperative period of transcatheter ASD closure [4]. In this article, we present the case of a 4-year-old girl who had a cryptogenic stroke secondary to a presumptive paradoxical embolism (PDE) after an unsuccessful attempt to close an ASD with a transcatheter procedure and in whom subsequent venous color Doppler showed deep venous thrombosis of the right lower extremity.

\section{Case Report}

A 4-year-old girl was referred for transcatheter closure of a secundum-type ASD at our catheter room because of recurrent upper respiratory tract infections. Her total body weight was $14.9 \mathrm{~kg}$, and her height was $96 \mathrm{~cm}$. Her cardiac examination was notable for a normal $\mathrm{S} 1$ and fixed splitting of S2 with a grade III/VI systolic ejection murmur heard at the left upper sternal border. Her electrocardiogram showed a sinus rhythm with an incomplete right bundle branch block. Chest X-ray showed mild cardiomegaly with increased pulmonary vascular markings. Transthoracic echocardiography demonstrated a secundum ASD measuring approximately $16.2 \mathrm{~mm}$ in diameter with left-toright shunting, right atrial and ventricular enlargement, and adequate rims that appeared to be suitable for percutaneous transcatheter closure (Fig. 1). After written informed consent was obtained from the patient's parents, a transcatheter ASD closure procedure was attempted with the patient under general anesthesia. Venous access was obtained from the right femoral veins using a $6 \mathrm{~F}$ sheath. At the start of the procedure, a bolus of weight-adjusted heparin (100 IU $/ \mathrm{kg}$ ) was administered intravenously. Cardiac catheterization showed mean right atrial pressure of $7 \mathrm{mmHg}$ with "A" wave of $12 \mathrm{mmHg}$ and "V" wave of $10 \mathrm{mmHg}$ and main pulmonary artery pressure of $29 / 10 \mathrm{mmHg}$. Oximetry 
Fig. 1 Transthoracic echocardiography demonstrated a secundum ASD with left-toright shunting
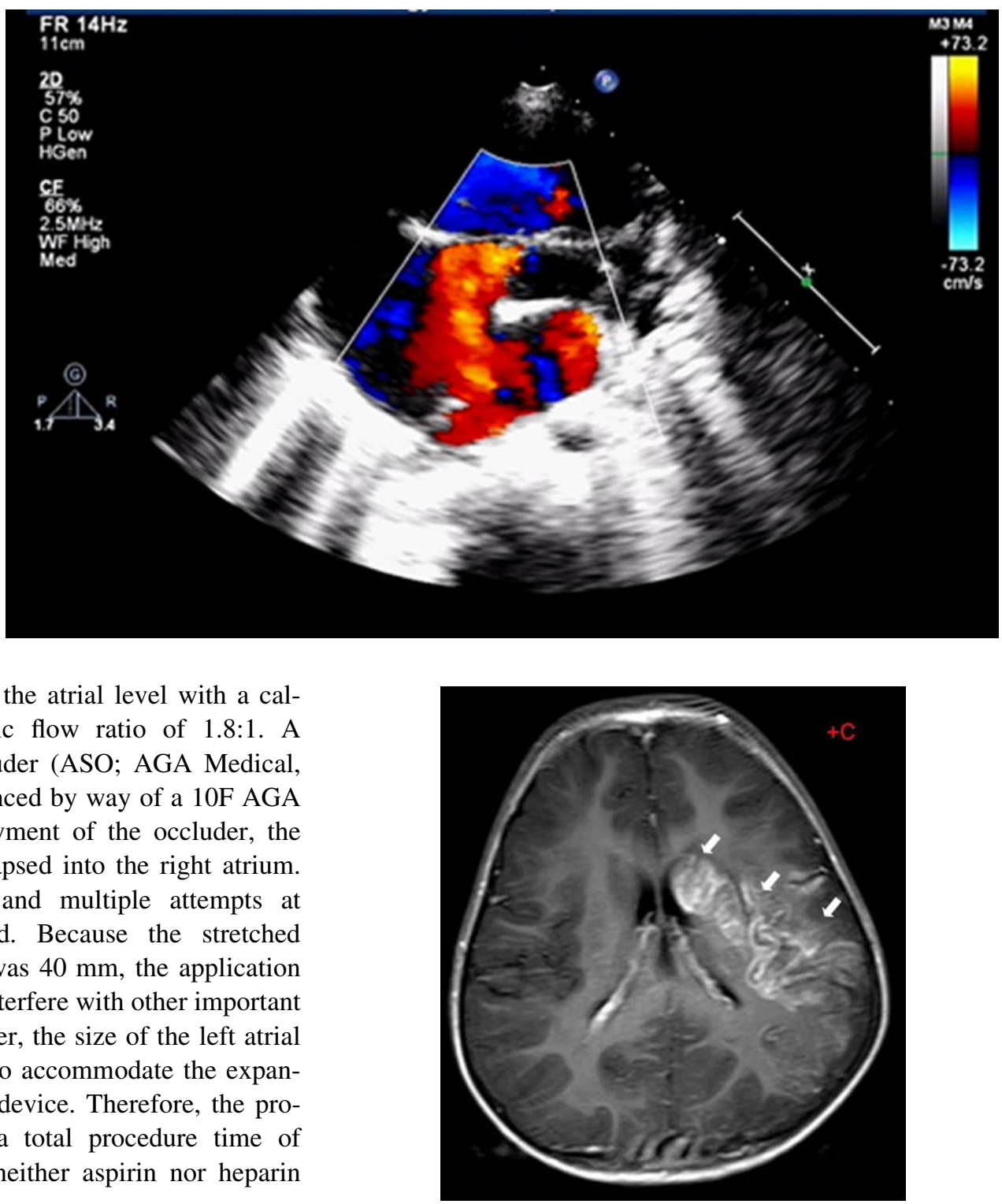

Fig. 2 Brain MRI showed acute cerebral infarctions in the leftward hippocampus, basal ganglia, and temporal lobe area (arrows)

On the basis of these findings, PDE was thought to be responsible for the systemic neurological deficits. The patient was anticoagulated with titrated doses of warfarin and commenced aspirin at $50 \mathrm{mg}$ daily. Repeat venous Doppler examination of the right lower extremity performed 15 days later showed no residual thrombus in the right femoral vein. Surgical closure of the ASD was strongly recommended for secondary prevention of recurrent systemic embolization; however, the patient's parents refused to give consent for the procedure. The patient was then discharged to a rehabilitation facility on oral anticoagulation. She has received intensive physical therapy, but further rehabilitation and recovery has been slow and unfavorable. 
Fig. 3 Venous color Doppler of the lower extremities showed DVT of the right femoral vein (arrow)

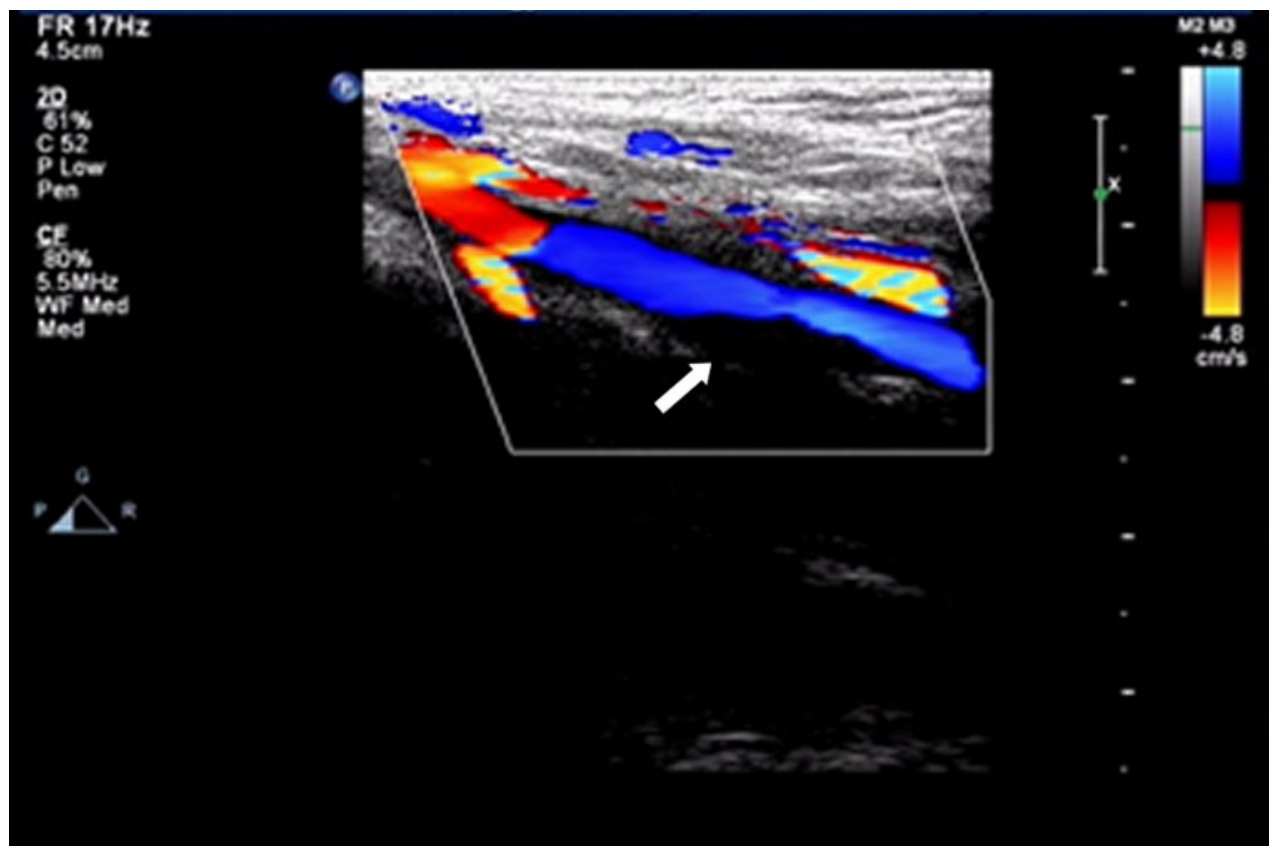

\section{Discussion}

The term "PDE" was coined by Cohnheim in 1877 [5] to describe a condition in which emboli of venous system origin enter the systemic arterial circulation by traversing through an abnormal communication between the right and left heart, leading to subsequent systemic embolic events. PDE is an uncommon but clinically important entity. In recent years, PDE has been recognized increasingly as a potential cause of cryptogenic stroke, particularly in younger patients [12].

Most cases of PDE are associated with a patent foreman ovale; less commonly, ASD, pulmonary arteriovenous malformation, and other intracardiac septal defects are responsible for PDE [3]. The pathophysiology is believed to be a transient increase in right heart pressure precipitating right-to-left shunting and thus allowing a venous thromboembolus to enter the arterial circulation [12]. Pulmonary embolism has been implicated as the most common cause of transient increase in right atrial pressure and right-to-left shunting in patients with an interatrial connection [9]. Valsalva maneuver, coughing, or straining are important physiological causes of a sudden increase of right atrial pressure and right-to-left shunting and have been demonstrated to be associated with $15 \%$ of PDEs [2, 3]. Johnson [7] proposed that PDE can be presumed in the presence of the following triad: (1) evidence of systemic arterial embolization in the absence of an embolic source in the left heart, (2) detection of an embolic source in the venous system, and (3) demonstration of an abnormal communication between the venous and arterial systems with a pressure gradient conducive to right-to-left shunting.
In the present case, the patient fulfilled all of the three criteria for diagnosis of presumptive PDE. We postulate that in our patient, an acute physiological increase in right atrial pressure, such as might be expected to occur during coughing or crying, probably caused transient right-to-left shunting, thereby allowing the venous thrombus to enter the left atrium. Systemic neurological sequalae then occurred.

It is worth noting that one of the major determinants of PDE that occurred in our patient is the presence of femoral deep venous thrombosis (DVT). Previous studies have demonstrated that catheterization by way of femoral vein cannulation is associated with a relatively high incidence of iliofemoral DVT. Using venous Doppler ultrasound, Davutoglu et al. [6], identified a $62.5 \%$ incidence of occult femoral DVT during uncomplicated routine femoral venous catheterization in patients undergoing electrophysiological studies. Talbott et al. [11], also using duplex Doppler ultrasonography, reported that 7 of 20 pediatric patients who underwent percutaneous femoral venous catheterization subsequently developed a femoral DVT, an incidence of $35 \%$. Importantly, the investigators found that in 6 of these 7 patients, femoral DVT was clinically silent and lacked signs when first demonstrated by ultrasonography. Abdullah et al. [1] demonstrated that the likelihood of developing catheter-related DVT was associated with mechanical factors, including the number of attempts at venipuncture, the number of catheters inserted, the material and diameter of catheter used, and the presence of a hypercoagulable state.

We believe that several underlying factors predisposed our patient to the development of femoral DVT: 
1. Our patient did not receive anticoagulation after the procedure

2. The use of a $10 \mathrm{~F}$ delivery sheath might have injured the vascular intima above the puncture site, leading to increased risk of local thrombosis

3. A tight pressure dressing on the venous puncture site may have impeded venous return, thus leading to a degree of venous stasis in the right leg. This abnormal venous flow pattern may have facilitated the development of DVT

4. A relative volume depletion or dehydration caused by fasting during the periprocedural period may have contributed to high blood viscosity

The present case highlights important clinical considerations: (1) In younger individuals presenting with cryptogenic stroke, clinicians should have a high index of suspicion for PDE. (2) Femoral DVT is a common complication of cardiac catheterization in children but usually has been overlooked. Moreover, femoral DVT is an important embolic source of PDE; thus, physicians should be aware of the risk of femoral DVT after cardiac catheterization. Based on our experience and the literature review, we make some recommendations that may be helpful to prevent or minimize the risk of DVT and subsequent PDE in similar cases.

\section{Recommendations}

1. Short-term, efficient anticoagulation and antiplatelet therapy should be administrated after cardiac catheterization, even if the procedure is unsuccessful

2. In younger patients undergoing cardiac catheterization by way of the femoral route, clinicians should be attentive to follow-up and consider routine venous ultrasound screening of the lower extremity after catheter removal, regardless of whether or not clinical signs of venous thrombosis are observed

3. Once a femoral DVT is detected, intensive anticoagulation treatment should be commenced as soon as possible. Insertion of an inferior vena cava filter to prevent the thrombotic material from escaping to the arterial circulation is a reasonable precaution
4. In younger patients with ASD who have a cryptogenic stroke, closure of the ASD is strongly recommended for the prevention of recurrent events

\section{Conflict of interest None declared.}

Open Access This article is distributed under the terms of the Creative Commons Attribution Noncommercial License which permits any noncommercial use, distribution, and reproduction in any medium, provided the original author(s) and source are credited.

\section{References}

1. Abdullah BJ, Mohammad N, Sangkar JV et al (2005) Incidence of upper limb venous thrombosis associated with peripherally inserted central catheters (PICC). Br J Radiol 78:596-600

2. Aburahma AF, Downham L (1996) The role of paradoxical arterial emboli of the extremities. Am J Surg 172:214-217

3. Ahmed S, Sadiq A, Siddiqui AK et al (2003) Paradoxical arterial emboli causing acute limb ischemia in a patient with essential thrombocytosis. Am J Med Sci 326:156-158

4. Chessa M, Carminati M, Butera G et al (2002) Early and late complications associated with transcatheter occlusion of secundum atrial septal defect. J Am Coll Cardiol 39:1061-1065

5. Cohnheim J (1877) Thrombose und Embolie. Vorlesungen über allgemeine pathologie. Hirschwald, Berlin, p 134

6. Davutoglu V, Kervancioglu S, Dinckal H et al (2004) High incidence of occult femoral vein thrombosis related to multiple venous sheaths during electrophysiological studies. Heart 90: 1061-1062

7. Johnson BI (1951) Paradoxical embolism. J Clin Pathol 4: 316-332

8. Lopez K, Dalvi BV, Balzer D et al (2005) Transcatheter closure of large secundum atrial septal defects using the $40 \mathrm{~mm} \mathrm{Am-}$ platzer septal occluder: results of an international registry. Catheter Cardiovasc Interv 66:580-584

9. Mirarchi FL, Hecker J, Kramer CM (2000) Pulmonary embolism complicated by patent foramen ovale and paradoxical embolisation. J Emerg Med 19:27-30

10. Rossi RI, Cardoso Cde O, Machado PR et al (2008) Transcatheter closure of atrial septal defect with Amplatzer device in children aged less than 10 years old: immediate and late follow-up. Catheter Cardiovasc Interv 71:231-236

11. Talbott GA, Winters WD, Bratton SL et al (1995) A prospective study of femoral catheter-related thrombosis in children. Arch Pediatr Adolesc Med 149:288-291

12. Wu LA, Malouf JF, Dearani JA et al (2004) Patent foramen ovale in cryptogenic stroke: current understanding and management options. Arch Intern Med 164:950-956 